$\begin{array}{cc}\text { ACADEMIA ROMÂNĂ } & \text { Rev. Roum. Chim., } \\ \text { 2020, 65(3), 277-281 }\end{array}$

\title{
PREPARATION AND EVALUATION OF PHOTOCATALYTIC ACTIVITY OF TIN-DOPED TITANIUM HYDROGEN PHOSPHATE FOR THE REMOVAL OF METHYL RED DYE
}

\author{
Mohamad KASSEM \\ Atomic Energy Commission of Syria. Chemistry Department P.O.Box 6091-Damascus. Syria
}

Received January 14, 2020

This study involves the preparation of titanium hydrogen phosphate $\alpha$ - $\mathrm{Ti}\left(\mathrm{HPO}_{4}\right)_{2} \cdot \mathrm{H}_{2} \mathrm{O} \quad(\alpha-\mathrm{TiP})$ and its tin-doped compounds for use as catalysts in the photolytic degradation of organic pollutants such as methyl red dye. The structural and textural properties of prepared samples were examined using various techniques: X-ray diffraction (XRD), Fourier-transform infrared spectroscopy (FTIR), nitrogen absorption and spectrophotometer. The results showed that the higher the amount of tin added, the lower crystallinity and the greater the surface area and pore volume of $\alpha$-TiP. These changes in the structural and textural properties of ( $\alpha$-TiP) had a significant effect on the photocatalytic degradation of the methyl red dye, where the degradation rate increased significantly by increasing the amount of tin.

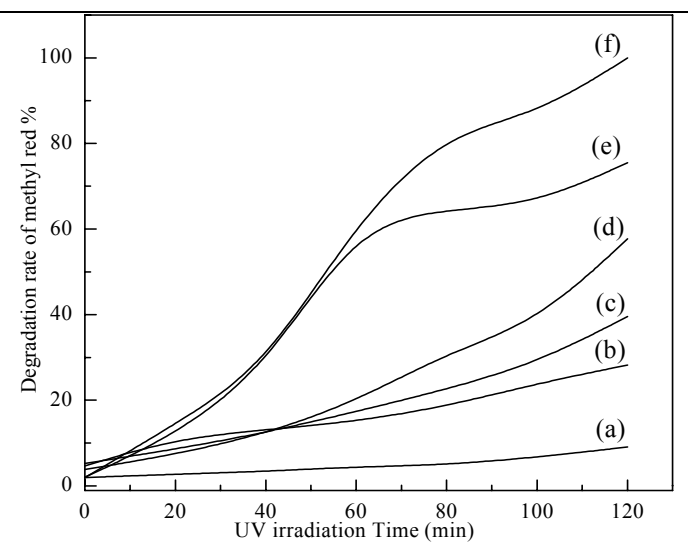

\section{INTRODUCTION}

Obtaining clean water has recently become one of the most important demands of life for all living organisms due to the presence of many types of pollutants such as dyes and organic compounds introduced by different industrial facilities. ${ }^{1,2}$. Various methods have been used to remove these pollutants. The most important one is photocatalytic destruction which has several features that make it the best choice for most researchers around the world such as high efficiency, speed in implementation and low cost. ${ }^{3-5}$.

A complete and fast photocatalytic process to destroy organic pollutants requires the use of catalysts with unique characteristics such as high surface area, physical and chemical stability, low cost, availability, non-toxicity, in addition to the appropriate electronic and optical properties suitable for this process. ${ }^{6-8}$. Within this approach, a variety of catalysts have been used, such as titanium oxide, zinc oxide and some other composites that achieve the above-mentioned features.

Researchers are still looking for new catalysts, either by innovation or by modifying some preexisting materials through doping, heating or grinding processes to make them more convenient for the photocatalytic process. $^{9-11 .}$

Tetravalent metals hydrogen phosphate $\mathrm{M}(\mathrm{IV})\left(\mathrm{HPO}_{4}\right)_{2} \cdot \mathrm{H}_{2} \mathrm{O}$ is an important class of inorganic substances that have recently attracted much attention for its multiple applications in ion exchange processes, intercalation, chemical catalysis, and molecular absorption. In addition, they have very remarkable physical and chemical properties. They are highly insoluble, thermally 
stable, chemically inert in neutral or acidic $\mathrm{pH}$ and capable of forming bonds with organic molecules. These specifications give attention to the use of these materials as photocatalysts either in pure form or after being doped by a metal element. ${ }^{12,13 .}$

This work aims to prepare $\alpha$-titanium hydrogen phosphate and their tin-doped compounds for using them as catalysts in the photocatalytic degradation process of methyl red in aqueous solutions.

\section{EXPERIMENTAL}

\section{Preparation of materials}

Titanium hydrogen phosphate $\alpha$-Ti( $\left(\mathrm{HPO}_{4}\right)_{2} \cdot \mathrm{H}_{2} \mathrm{O}(\alpha$-TiP) has been prepared according to the methods previously reported, ${ }^{14,15}$ briefly: a reaction mixture $\mathrm{TiCl}_{3}(15 \%)$,$\mathrm{H}_{3} \mathrm{PO}_{4}(85 \%)$, with constant $\mathrm{P}$ :Ti molar ratio $4: 1$ has been refluxed at a temperature of $100{ }^{\circ} \mathrm{C}$ for 24 hours. The precipitate was washed with distilled water until $\mathrm{PH}=7$ then dried at $60^{\circ} \mathrm{C}$.

Sn-doped $\alpha$-TiP samples were obtained by impregnation method where a fixed mass of pure material was suspended in a solution containing tin chloride (IV) so that the following molar ratios were achieved: $\mathrm{Sn} / \mathrm{Ti}=0,0.2,0.4,0.6,0.8$ and 1 . The resulting suspension was stirred for 24 hours, at room temperature, then filtered and washed several times with distilled water. The obtained solid was dried at $50^{\circ} \mathrm{C}$ for 24 hours.

\section{Characterization methods}

During the different phases of this work, a variety of techniques were used including X-ray diffraction (STADI P "STOE \& Cie GmbH", Germany), Fourier transform, infrared spectroscopy ("Thermo Nicolet 6700" USA), surface area analyzer (NOVA 2200 "Quantachrome Corp.", USA). For the evaluation the methyl red degradation, a UV-Vis spectro- photometer (AvaSpec-2028 “Avantes B.V.”, Holland) was used.

\section{RESULTS AND DISCUSSION}

\section{XRD Measurements}

X-ray diffraction measurements revealed, as shown in Fig. 1, that the $\alpha$-TiP prepared in this work is a high-quality polycrystalline material (Fig. 1a). All peaks displayed in this diffraction pattern are fully compatible with the JCPDS (card 80-1067).

The effect of tin addition in the $\alpha$-TiP structure is manifested through XRD patterns (Fig. 1bFig. 1f) as a decrease in peak intensity and an increase in their width as the proportion of tin rises. This dissimilarity could indicate the conversion of $\alpha$-TiP from the crystalline state to the semi-crystalline or low crystalline which can be attributed to a diminution in the crystallite size due to defects that may have formed as a result of the difference in phosphorous/metal ratio between the doped and pure $\alpha$-TiP samples.

The crystallite size was calculated using Scherer's equation: $t=0.9 \lambda /(B \cos \theta)$, the values of this parameter are listed in table 1 for pure and doped $\alpha$-TiP.

It can also be noted that all diffraction patterns related to doped specimens do not show additional peaks indicating the presence of phases other than $\alpha$-TiP.

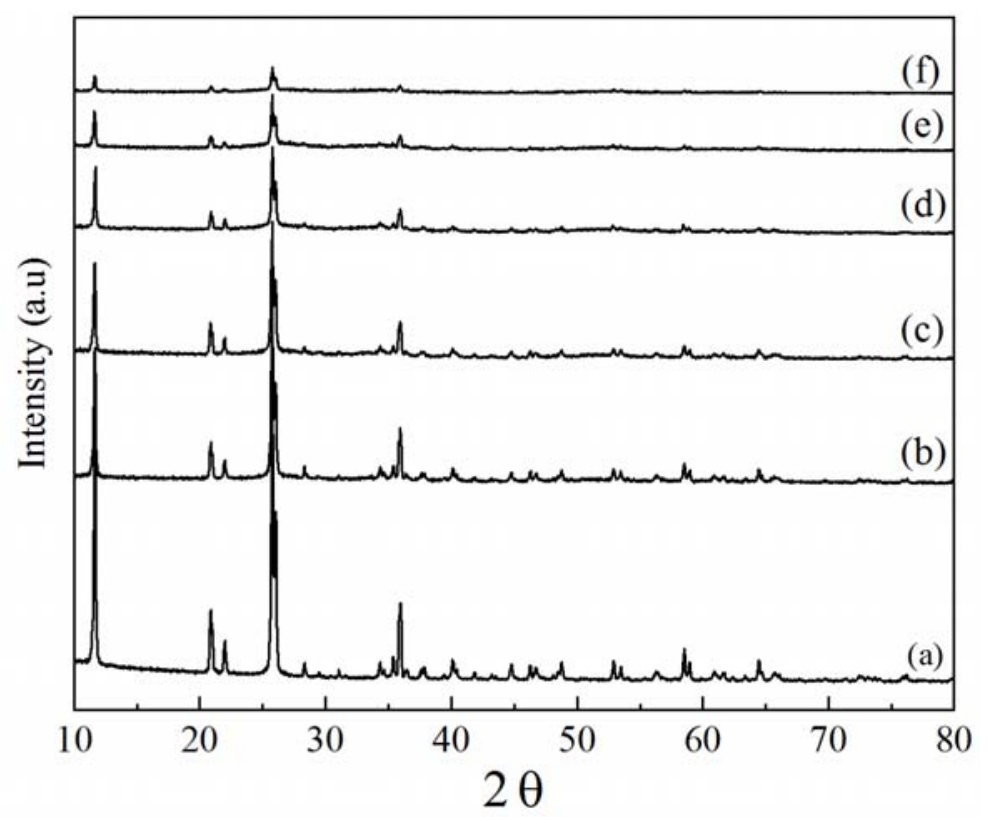

Fig. 1 - XRD patterns for pure $\alpha$-TiP (a) Sn-doped $\alpha$-TiP: $\mathrm{Sn} / \mathrm{Ti}=0.2(b) 0.4(c) 0.6(d) 0.8(e) 1(f)$. 


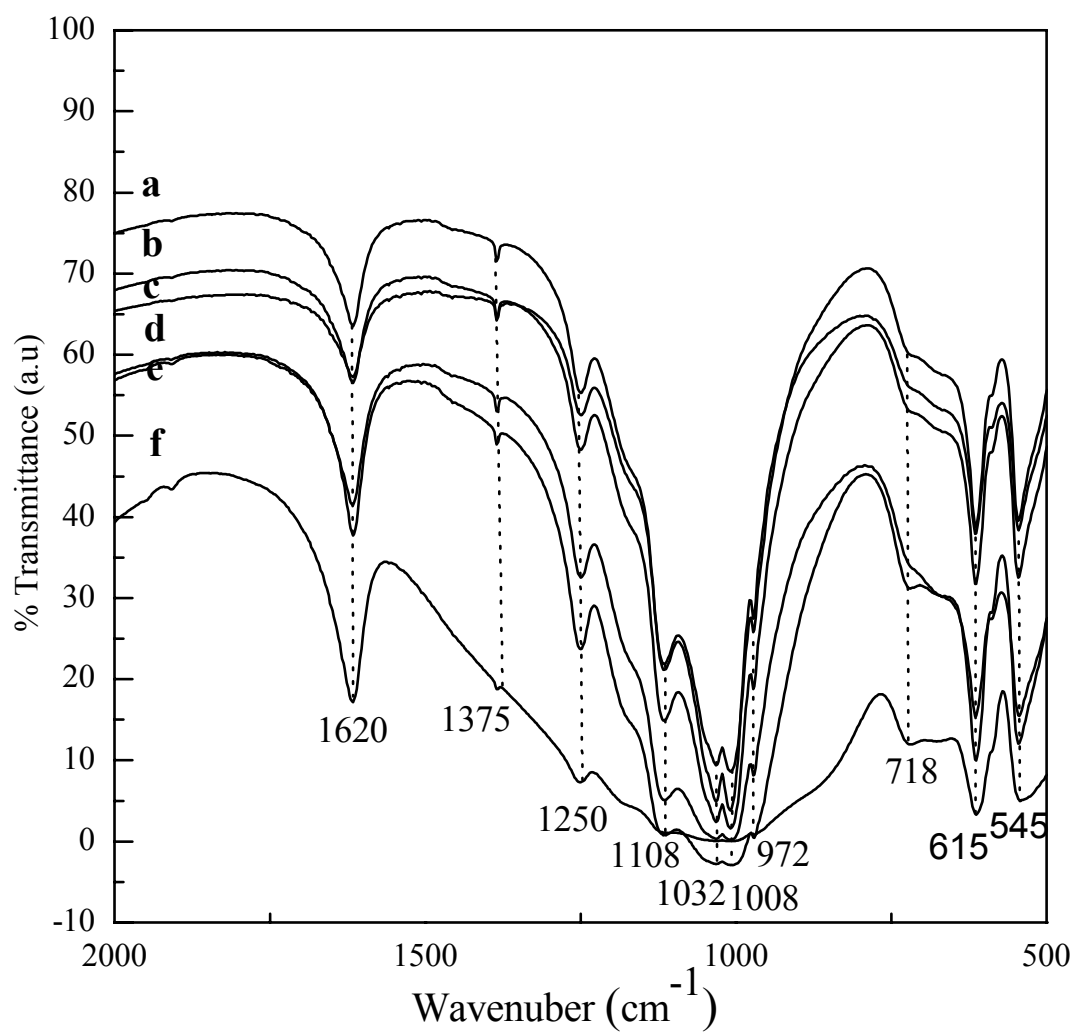

Fig. 2 - FT-IR spectra for pure $\alpha$-TiP (a) Sn-doped $\alpha$-TiP : Sn/Ti= 0.2 (b) 0.4 (c) 0.6 (d) 0.8 (e) 1 (f).

\section{Fourier transform infrared (FT-IR)}

To verify the data disclosed by X-ray diffraction, FTIR spectra were recorded for all samples as shown in Fig. 2. The spectrum related to the un-doped material (Fig. 2a) is in good accordance with that reported by other authors. ${ }^{16-}$ 18 .

For Sn-doped samples, FTIR spectra remain similar to the pure substance spectrum but with some changes becoming more and more evident as tin concentration increases. These alterations are reflected in the broadening of the bonds group located in the wavelength range between 800 and $1300 \mathrm{~cm}^{-1}$ which includes P-O-P $\left(972 \mathrm{~cm}^{-1}\right)$, P-O $\left(1008 \mathrm{~cm}^{-1}\right)$, HPO4 ${ }^{-2}\left(1032 \mathrm{~cm}^{-1}\right)$, Ti-O-P (1055 $\left.\mathrm{cm}^{-1}\right)$ and $\mathrm{P}=\mathrm{O}\left(1250 \mathrm{~cm}^{-1}\right)$. Such spectral broadening can be associated with deformation of the chemical environment surrounding these bonds induced by the addition of tin, which may explain the decrease in the degree of crystallization of $\alpha$ TiP observed in XRD results.

\section{N2 adsorption-desorption measurements}

To explore further effects of tin addition on $\alpha$ - titanium phosphate, the textural characteristics
(Surface area, Pore volume, Pore size) were estimated for the investigated samples. These are very important factors for the suitability of the material to be used as a photocatalyst ${ }^{19,20}$. They have been determined through nitrogen adsorptiondesorption measurement at liquid N2 temperature in a relative pressure range of $0.05-0.95$. The values of these charactestics, listed in Table 1, disclose a good correlation with the tin concentration. For example, samples with a high concentration of tin have a larger surface area and a smaller pore diameter.

These results are also consistent with those obtained by XRD, where samples with a larger surface area have a smaller crystallite size.

\section{Photocatalytic Activity}

The photocatalytic activity of pure and Sndoped $\alpha$-TiP materials was investigated by applying a heterogeneous photocatalytic process for the degradation of methyl red dye in the presence of ultraviolet radiation. The degradation rate was estimated by measuring the concentration of dye in the solution before and after irradiation according to the following equation:

$$
\text { Degradation rate }(\%)=\left(\left(\mathrm{C}_{0}-\mathrm{C}\right) / \mathrm{C}_{0}\right) \times 100
$$


Table 1

Crystallite size and textural properties for $\mathrm{Sn}$-doped $\alpha$-TiP samples $0<\mathrm{Sn} / \mathrm{Ti}<1$

\begin{tabular}{ccccc}
\hline $\begin{array}{c}\text { Sn/Ti } \\
\text { Molar ratio }\end{array}$ & $\begin{array}{c}\text { SSA } \\
\left(\mathbf{m}^{2} / \mathbf{g}\right)\end{array}$ & $\begin{array}{c}\text { Total pore } \\
\text { volume } \\
(\mathbf{m L} / \mathbf{g})\end{array}$ & $\begin{array}{c}\text { Crystallite } \\
\mathbf{s i z e} \\
\mathbf{( n m )}\end{array}$ & $\begin{array}{c}\text { Pore } \\
\text { diameter } \\
\mathbf{( n m )}\end{array}$ \\
\hline 0 & 4.5 & 0.0048 & 1.2 & 4.3 \\
0.2 & 38.1 & 0.0351 & 1.2 & 3.7 \\
0.4 & 65.2 & 0.0544 & 1.2 & 3.3 \\
0.6 & 96.4 & 0.0715 & 1.2 & 3.0 \\
0.8 & 110.7 & 0.0803 & 1.1 & 2.9 \\
1 & 116 & 0.0876 & 1.0 & 2.5 \\
\hline
\end{tabular}

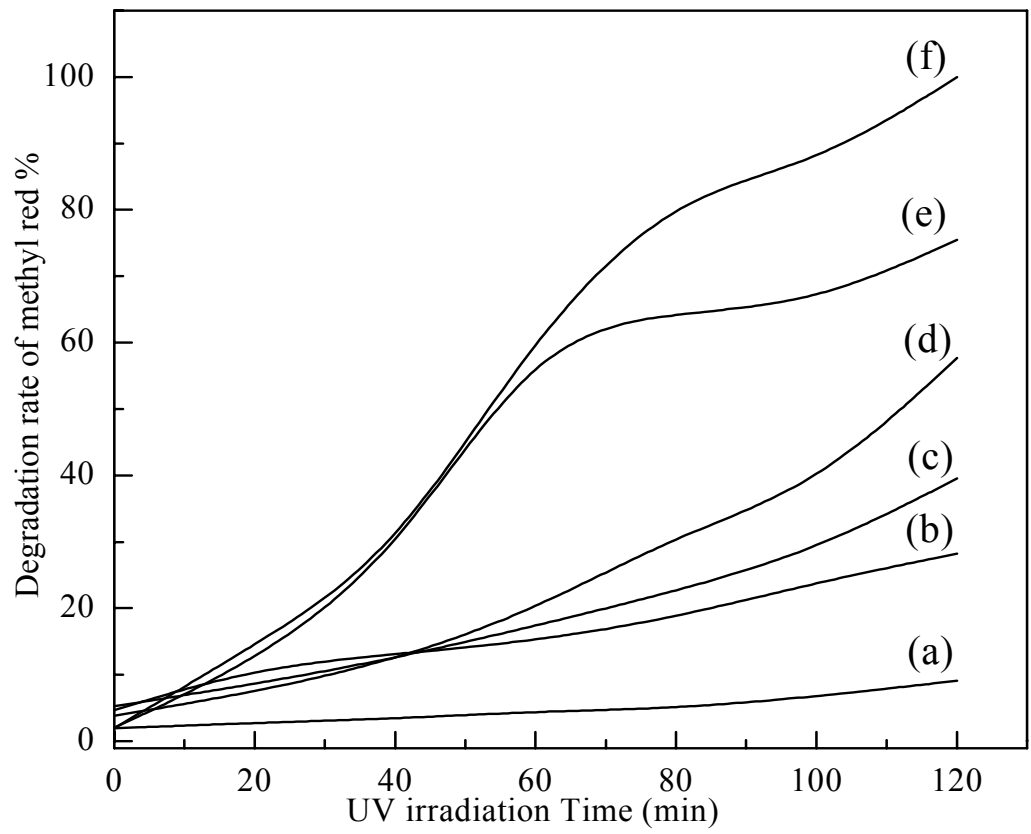

Fig. 3 - Photocatalytic degradation of methyl red dye on pure $\alpha$-TiP (a) Sn-doped $\alpha$-TiP : Sn/Ti= 0.2 (b) 0.4 (c) 0.6 (d) 0.8 (e) 1 (f).

The results obtained, as shown in Fig. 3, indicate that the photocatalytic activity of pure $\alpha$-TiP remains low during the entire irradiation period $0-120 \mathrm{~min}$ (Fig. 3a). For Sn-doped $\alpha$-TiP, the degradation of methyl red increase slowly during the irradiation period, in the case of samples containing $(\mathrm{Sn} / \mathrm{Ti})$ ratios between $0-0.6$, where it does not exceed $15 \%$, in the first hour, and about $55 \%$ at the end of the second one (Fig. 3a-3d).

Regarding the samples with $(\mathrm{Sn} / \mathrm{Ti})$ ratios greater than 0.6 (Fig. 3e-3f), the degradation begins to increase rapidly after 20 minutes of irradiation and reaches about $60 \%$ in the first hour and about $98 \%$ at the end of the second for samples containing a high concentration of tin $(\mathrm{Sn} / \mathrm{Ti}=1)$.

The differentiation in the photocatalytic activity between pure and sn-doped $\alpha$-TiP can be attributed to several changes caused by the addition of tin, the most important being a rise in the specific surface area of the samples, that increase the number of dye molecules adsorbed on the surface of the catalyst and thus the enhancement of photocatalytic activity. In addition, the presence of tin may alter the energy gap of $\alpha$-TiP, another factor that helps also to raise the catalytic activity.

\section{CONCLUSION}

The $\alpha$-titanium phosphate prepared in this work is a well crystallized material.

The impregnation method is a good way to prepare $\alpha$-titanium phosphate doped with different concentrations of tin.

The amount of tin added to $\alpha$-TiP had a noticeable effect on the crystallization degree and 
textural properties (surface area, pore size) which in turn altered the photocatalytic activity of investigated materials.

The degradation rate of methyl red has increased more rapidly when samples with a higher $\mathrm{Sn} / \mathrm{Ti}$ ratio have been used as photocatalysts.

Acknowledgements: The author would like to thank Professor. I. Othman, Director General, and Dr. A. Allaf, head of the chemistry department for their encouragement. Thanks are also to Mr. H. Harmalani for assistance in the experimental works as well as Ms. Z. Hariri, Mr. T. Jeejan and Mr. A. Alzeer for the spectroscopic measurements

\section{REFERENCES}

1. I. M. Sujaul, M. A. Hossain, M. A. Nasly and Md. A. Sobahan, Am. J. Environ. Sci., 2013, 9, 120-129.

2. R. Kant, Nat. Sci., 2012, 4, 22-26.

3. A. A. Al-Kahtani, J. Biomater. Nanobiotechnol., 2017, 8, 66-82.

4. T. W. Gebreslassie, M. Pattabi and R. M. Pattabi, Inter. J. Sci. Res., 2015, 4, 2252-2264.

5. T. K. Mandal, S. P. K. Malhotra and R. K. Singha, Roman. J. Mater., 2018, 48, 32 -38.

6. Y. H.Chiu, T. F. M. Chang, C. Y. Chen, M. Sone and Y. J. Hsu, Catal., 2019, 9, 430-462.

7. J. Ge, Y. Zhang and S. J. Park, Mater., 2019, 12, 19161942.
8. S. Mortazavian, A. Saber and D. E. James, Catal., 2019, 9, 360-380.

9. M. Aminia and M. Ashrafia, Nano. Chem. Res., 2016, 1, 9-86.

10. P. Gonçalves, R. Bertholdo, J. A. Diasb, S. C. Maestrelli and T. R. Giraldi, Mater.Res., 2017, 20, 181-189.

11. D. R. Shinde, P. S.Tambade, M. G. Chaskar and K. M. Gadave, Drink. Water. Eng. Sci., 2017, 10, 109-117.

12. P. Olivera-Pastor, P. Maireles-Torres, E. RodríguezCastellón, A. Jiménez-López, T. Cassagneau, D. J. Jones and J. Rozière, Chem. Mater., 1996, 8, 1758-1769.

13. R. Lin and Y. Ding, Mater., 2013, 6, 217-243.

14. H. B. Ortíz-Oliveros, R. Ma. Flores-Espinosa, E. Ordoñez-Regil and S. M. Fernández-Valverde, Chem. Eng. J., 2014, 236, 398-405.

15. S. Bruque, M. A. G. Aranda, E. R. Losilla, P. OliveraPastor and P. Maireles-Torres, Inorg. Chem., 1995, 34, 893-899.

16. A. Attia, Q. Wang, Xi. Huang and Y. Yang, J. Solid. State. Electrochem., 2012, 16, 1461-1471.

17. B. B. Sahu and K. Parida, J. Colloid. Interface. Sci., 2002, 248, 221-230.

18. W. Janusz, S. Khalameida, E. Skwarek, J. SkubiszewskaZie, ba, V. Sydorchuk and B. Charmas, J. Therm. Anal. Calorim., 2018, 16,

19. D. J. Jones, G. Aptel, M. Brandhorst, M. Jacquin, J. JimeÂnez-JimeÂnez, A. JimeÂnez-LoÂpez, P. MairelesTorres, I. Piwonski, E. RodrõÂguez-CastelloÂn, J. Zajaca and J. RozieÁre, J. Mater. Chem., 2000, 10, 19571963.

20. B. Guo, H. Shen, K. Shu, Y. Zeng and W. Ning, J. Chem. Sci. 2009, 121, 317-321. 
